NASA Technical Memorandum 87231

\title{
Computational Engine Structural Analysis
}

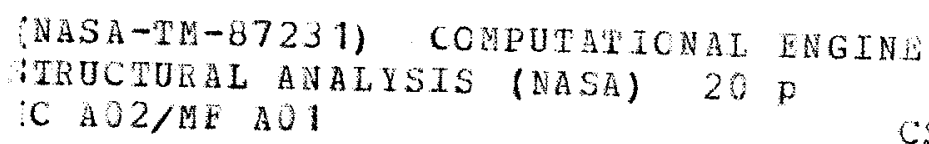

\author{
$63 / 39$ Unclas
}

Christos C. Chamis and Robert H. Johns

Lewis Research Center

Cleveland, Ohio

Prepared for the

31st International Gas Turbine Conference and Exhibit sponsored by the American Society of Mechanical Engineers Dusseldorf, West Germany, June 8-12, 1986

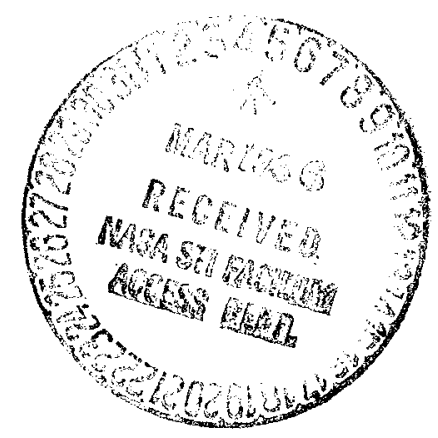


SUMMARY . . . . . . . . . . . . . . . . . . . . . . . . . 1 INTRODUCTION - LEWIS'S RESEARCH ACTIVITIES ON COMPUTATIONAL ENGINE STRUCTURAL ANALYSIS . . . . . . . . . . . . . . . 1 AEROELASTICITY OF BLADES ROTORS . . . . . . . . . . . . . . . . 2 HIGH VELOCITY IMPACT OF FAN BLADES . . . . . . . . . . . . . . . . 2 BLADE LOSS TRANSIENTS . . . . . . . . . . . . . . . . . . . . 2 ROTOR/STATOR/SQUEEZE-FILM-BEARING INTERACTION . . . . . . . . . . . . 3 BLADE-FRAGMENT/ROTOR-BURST CONTAINMENT . . . . . . . . . . . . . . . . . 4 ADVANCED TURBOPROP STRUCTURAL BEHAVIOR . . . . . . . . . . . . . . . 4 SUMMARY OF RESULTS . . . . . . . . . . . . . . . . . . . 5 REFERENCES ............................. 5 


\title{
COMPUTATIONAL ENGINE STRUCTURAL ANALYSIS
}

\author{
Christos C. Chamis and Robert H. Johns \\ National Aeronautics and Space Administration \\ Lewis Research Center \\ Cleveland, Ohio 44135 , U.S.A.
}

\begin{abstract}
SUMMARY
A significant research activity at the NASA Lewis Research Center is the computational simulation of complex multidisciplinary engine structural problems. This simulation is performed using computational engine structural analysis (CESA) which consists of integrated multidisciplinary computer codes in conjunction with computer post-processing for "problem-specific" application. A variety of the computational simulations of specific cases are described in some detail in this paper. These case studies include (1) aeroelastic behavior of bladed rotors, (2) high velocity impact of fan blades, (3) blade-loss transient response, (4) rotor/stator/squeeze-film/bearing interaction, (5) blade-fragment/rotor-burst containment, and (6) structural behavior

of advanced swept turboprops. These representative case studies were selected to demonstrate the breadth of the problems analyzed and the role of the computer including post-processing and graphical display of voluminous output data.
\end{abstract}

\section{INTRODUCTION}

The need for lightweight, durable, fuel-efficient, cost-competitive aircraft requires engine structures which are made from advanced materials including composites, and which resist higher temperatures, maintain tighter clearances and reduce maintenance costs. These requirements tend to increase the complexity of aircraft engines which are already intricate structural systems consisting of dynamically interacting components. Prediction of the integrated response of these systems requires complex analytical models (finite element, for example) as well as complex static, periodic, transient, and impact analyses. A major engine structures program (fig. 1) is currently being conducted by NASA Lewis, with emphasis on computational engine structural analysis (CESA). Several independent computer codes for specific aspects of engine structures modeling and/or structural analysis are being developed as part of this program. These codes include: (1) interactive finite elements for bearing/shaft interaction, (2) engine structural component modeling, (3) automatic finite element generators, (4) nonlinear analysis with various levels of sophistication, (5) nonlinear constitutive relationships, (6) automatic thermal load transfer, (7) blade-loss transient analysis, (8) aeroelastic analysis of bladed disks, (9) multimode impact analysis, and (10) structural tailoring of engine blades.

Several of these computer codes were briefly described in a previous paper (ref. 1). The objective of this paper is to illustrate typical computational results obtained from these codes for: (1) aeroelastic behavior, (2) highvelocity impact, (3) blade-loss transients, (4) rotor/stator/squeeze $\mathrm{film} /$ bearing interaction, (5) blade fragment containment, and (6) advanced turboprop structural behavior. The role of the computer in the solution of these 
complex multidisciplinary problems, the post-processing, and the graphic display of voluminous results obtained therefrom are emphasized.

\section{AEROELASTICITY}

The multidisciplinary analysis required for predicting aeroelastic behavior including flutter is performed by a special-purpose code (ref. 2). This code couples appropriate aerodynamics with the structural response of bladed, shrouded disks. Flutter is an important aeroelastic design consideration for fan blades. When it occurs it subjects a blade to high-amplitude cyclic loading which can rapidly degrade its structural integrity and lead to blade failure.

Blade failure induces rotor imbalance which, if of sufficient magnitude can cause rotor stage failure and/or bearing failures, both of which can lead to catastrophic engine fallure, if only very infrequentiy.

The computer code predicts the flutter speed and the blade motion near or at this speed. The code uses cyclic symmetry substructuring concepts and the widely-used NASTRAN structural analysis computer code. A computer plot of a typical sector of a bladed shrouded disk analyzed is shown in figure 2 . Computed graphical results of the motion of this sector near flutter speed are shown exaggerated in figure 3. The character of the motion and location of high bending (fatigue) stresses are readily observable in this figure.

\section{HIGH VELOCITY IMPACT OF FAN BLADES}

High-velocity impact which can result in foreign object damage (FOD) is an important design consideration in aircraft turbine engine fan blades. Some research activities at NASA Lewis focused on developing technology for impactresistant fan blades using advanced design concepts and several computer codes (ref. 1).

A computer-produced three-dimensional finite element model for one of these advanced blade concepts is shown in figure 4 . Stress contour plots obtained using this finite element model are shown in figure 5 for several blade depth locations or "surfaces." Impact location and blade root stresses obtained using modal synthesis for transient analysis are summarized in figure 6 .

The amount of information associated with high-velocity impact analysis is very voluminous. Computer post-processing and computer graphics including computer-produced movies is the practical way to handle it. Furthermore, computer color graphics enhance the interpretation of the output relative to displacement magnitudes, stress distributions and magnitudes, and transient motion of the impacted blade.

\section{BLADE-LOSS TRANSIENTS}

Partial or full blade loss due to impact, for example, causes stage imbalance which can induce rotor transient whirling. This type of imbalance 
and the resulting transient and steady-state motion must be predictable so that appropriate tolerances and operating restrictions can be provided. Prediction of blade loss transient response requires an integrated structural dynamic analysis where the bladed rotor, shaft, and bearings are all modeled in the analysis. A computer code (Turbine Engine Transient Response Analysis [TETRA] ref. 1) was developed for such an integrated analys is.

TETRA uses the component element analysis method with progressively reduced model complexity as shown in figure 7. TETRA output is also voluminous. Computer graphics is the practical way to display this type of output. Typically TETRA produces displacement/time history plots as shown in figure 8 , or corresponding frequency-domain plots as shown in figure 9 . Both of these plots are valuable and necessary in interpreting the blade loss transient response and in assessing the adequacy of the design.

\section{ROTOR/STATOR/SQUEEZE-F ILM/BEARING INTERACTION}

Squeeze-film damper bearings are designed for specified blade-1oss transients and rotor imbalance and vibration. Rotor/squeeze-film/bearing/ stator interactions are inherently nonlinear because of: (1) the inherent behavior characteristics of squeeze films, particularly during transient situations, and (2) potential structural interactions wherein either large deformation kinematics or material nonlinearity (plasticity) are excited.

Because of such behavior, the overall rotor-bearing-stator computational simulation must be able to incorporate the various sources of nonlinearity. Additionally, to enable efficient solutions in situations where in the behavior of the model components is linear, the overall simulation scheme must incorporate substructuring capabilities. Furthermore, since transient problems need to be considered, such features must be accommodated by the various integration algorithms used to solve the governing model equations.

A general purpose squeeze-film-damper/interactive-force finite element model has been developed as part of the NASA Lewis program (refs. 3 to 4 ). This finite element model was implanted in a general purpose finite element computer code. Three different numerical integration methods were also incorporated to solve the governing structural dynamics equations of the interactive finite element. The general purpose code is used subsequently to predict the structural dynamic response of the rotor/stator coupled structure subjected to imbalance and impulse type excitations. The structural dynamic responses predicted include: (1) bearing/rotor trajectories, (2) stator trajectory, (3) rotor orbit, and (4) force, velocity and acceleration histories at a given location in the coupled structure. Three-dimensional post-processors are used to graphically display the predicted results in isometric views. Typical computer post-processing results are shown in figure $10 \mathrm{for}$ a shaft/single bearing interaction, and in figure 11 for a shaft/multibearing interaction. These graphical results clearly indicate squeeze film pressures, orbit motion, orbit stability, clearances, and respective margins. 


\section{BLADE-FRAGMENT/ROTOR-BURST CONTAINMENT}

Blade-fragment containment and rotar-burst containment are critical design requirements for turbine engines for commercial aircraft. The computational simulation of blade-fragment and/or rotor-burst containment require complex analyses including fragment tracking, nonlinear material behavior, and prediction of finite strains, large rotations and large displacements. These complex analyses were developed as part of the NASA Lewis program and incorporated into an integrated computer code using nonlinear finite element theory (ref. 5).

The containment computational simulation using this code starts with the initial configuration of the containment ring, rotor speed, fragment size, and fragment location. The simulation proceeds to determine the fragment kinetic energy, momentum, direction, and impact point on the containment ring. The transient structural response of the ring from this contact point is tracked as a function of time. The transient structural response generally includes displacements, strains and stresses.

Because of the importance of fragment containment, extensive experimental studies have been conducted on specific rotors and containment rings. The computational simulation results for one of these cases are included herein. The containment ring geometry and properties and the rotor characteristics are shown in figure 12. A computer plot of the shape of the deformed ring at about its maximum deformation is shown in figure 13. Computer plots for the stresses at the corresponding time are shown in figure 14 for the outer surface and in figure 15 for the inner surface.

\section{ADVANCED PROPELLER STRUCTURAL BEHAVIOR}

The use of thin, highly swept, twisted propeller blades is important to the development of fuel-efficient and quiet advanced aircraft. High sweep angles produce significant reductions in noise and are therefore a desirable design feature. However, blades of this type (thin, highly swept, twisted) exhibit complex structural response under a centrifugal force field, which requires special analysis techniques for accurate characterization. These techniques are required to establish the structural dynamic response of the turboprop blades at operating rotor speeds including the avoidance of possible leading edge buckling under centrifugal loads. Computational simulation studies (refs. 6 to 8 ) were performed at NASA Lewis to determine analytically the structural behavior and possible leading edge buckling of advanced, highly swept turboprop airfoils in centrifugal force fields. Computational simulation studies were also performed in order to identify any advantages of using "high performance" composites in order to change the regions of instability. The theoretical studies were performed using an in-house program (COBSTRAN, ref. 1) designed for composite blade analysis. Schematics of the rotor, the propeller and the finite element model used in the analyses are shown in figure 16.

Computer output results for rotor speed effects on the vibration mode shape and potential for leading edge buckling are shown in figure 17 . Computerplotted mode shapes of the propeller blade superimposed on a Campbell Diagram are shown in figure 18. Display of the amount of computer output information summarized in figure 18 would be impractical without computer post-processing and graphical display. The avallability of all this information in one figure 
makes it convenient to assess the adequacy of the design on a quantitative basis.

\section{SUMMARY OF RESULTS}

The complex behavior of integrated aircraft engine structures requires the solution of interacting multidisciplinary problems. Some recent research activities at NASA Lewis focus on computational engine structural analysis (CESA). CESA is essentially the computational simulation of complex engine structural behavior using multidisciplinary integrated computer programs in conjunction with extensive computer post-processing and graphical display of computer output. Representative case studies were selected and are described in some detail to illustrate the role of the computer on the computational simulation of complex multidisciplinary engine structural problems. These case studies include: (1) aeroelastic behavior of bladed rotors, (2) high velocity impact of fan blades, (3) blade-loss transient response, (4) rotor/ stator/squeeze-film/bearing interaction, (5) blade-fragment/rotor-burst containment, and (6) structural behavior of advanced swept turboprops. These representative case studies further demonstrate the indispensable utility of computer post-processing and graphical display of voluminous analysis results which make it possible to readily assess the adequacy of a design on a quanti. tative basis.

\section{REFERENCES}

1. Chamis, C.C., "Integrated Analys is of Engine Structures,: NASA TM-827i3, 1981.

2. Smith, G.C.C. and Elchuri, V., "Aeroleastic and Dynamic Finite Element Analyses of a Blader Shrouded Disk," Textron Bell Aerospace Co., Buffalo, NY, 1980. (NASA CR-159728)

3. Adams, M.L., Padovan, J., and Fertis, D.G., "Finite Element for Rotor/ Stator Interactive Forces in General Engine Dynamic Simulation," EDA-201-3A, Akron Univ., Akron, OH, 1980. (NASA CR-165214)

4. Padovan, J., et al., "Engine Dynamic Analysis, with General Nonlinear Finite Element Codes," Akron Univ., Akron, OH, 1982. (NASA CR-167944)

5. Roda1, J.J.A. and Witmer, E.A., "Finite-Strain Large-Deflection ElasticViscoplastic Finite-Element Transient Response Analysis of Structures," ASRL-TR-154-15, Massachusetts Institute of Technology, Cambridge, MA, 1979. NASA CR-159874)

6. Aiello, R.A. and Chamis, C.C., "Large Displacement and Stability Analysis of Noni inear Propeller Structures, "Tenth NASTRAN User's Colloquium, NASA CP-2249, 1982, pp. 112-132.

7. Chamis, C.C. and Aiello, R.A., Tensile Buckling of Advanced Turboprops," Journal of Aircraft, Vol. 20, No. 11, Nov. 1983, pp. 907-912.

8. Hirschbein, M.S., et al., "Structural and Aeroelastic Analys is of the SR-7L Propfan," NASA TM-86877, 1985. 


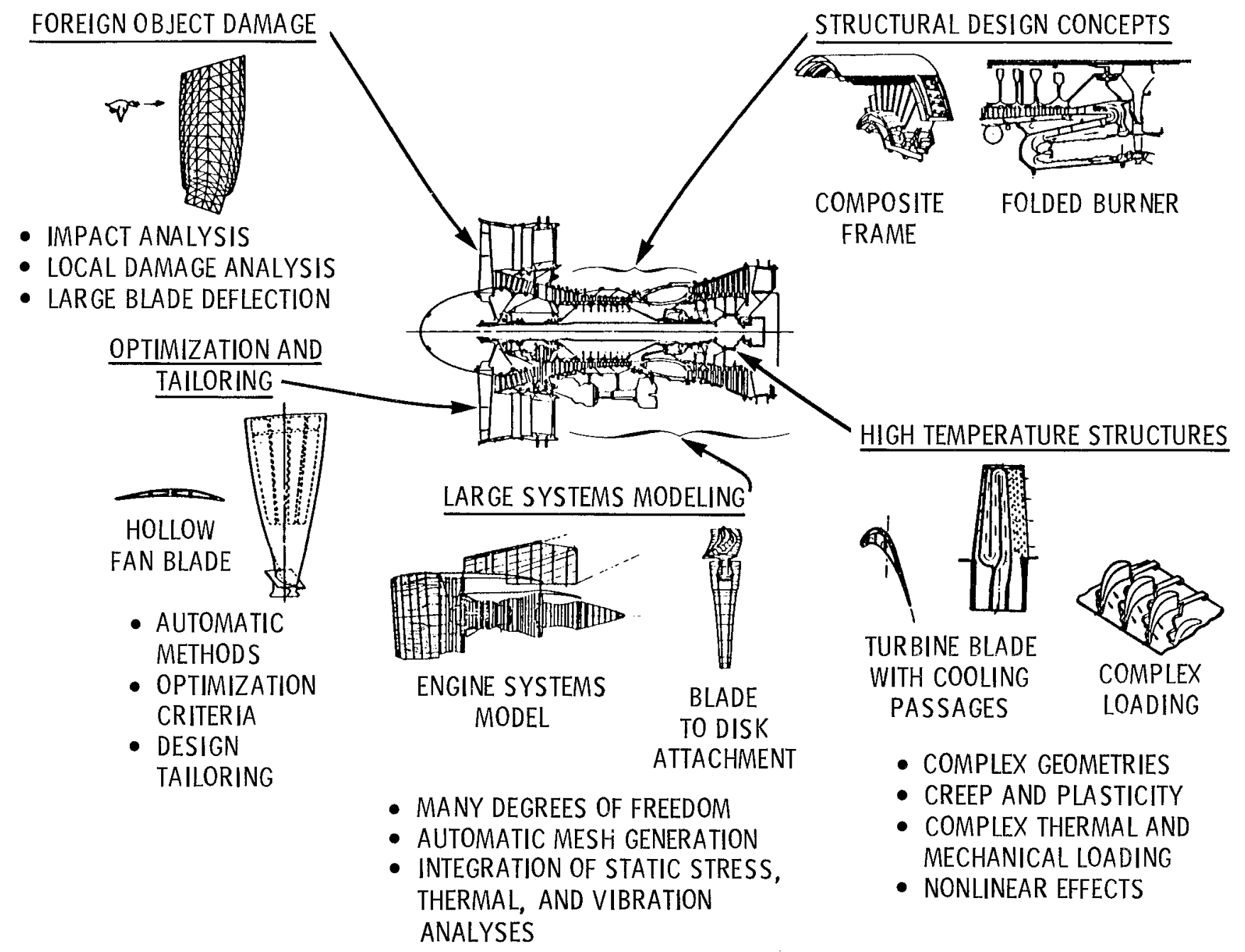

Figure 1. - Engine structural components amenable to computational simulation. 


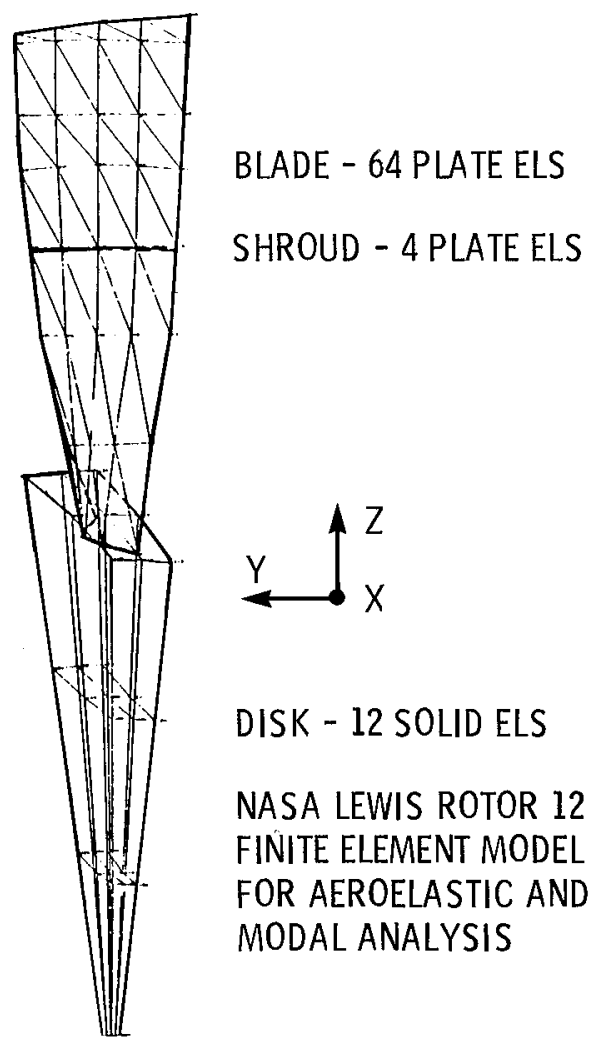

Figure 2. - Finite element model for aeroelastic analysis of bladed shrouded disks.
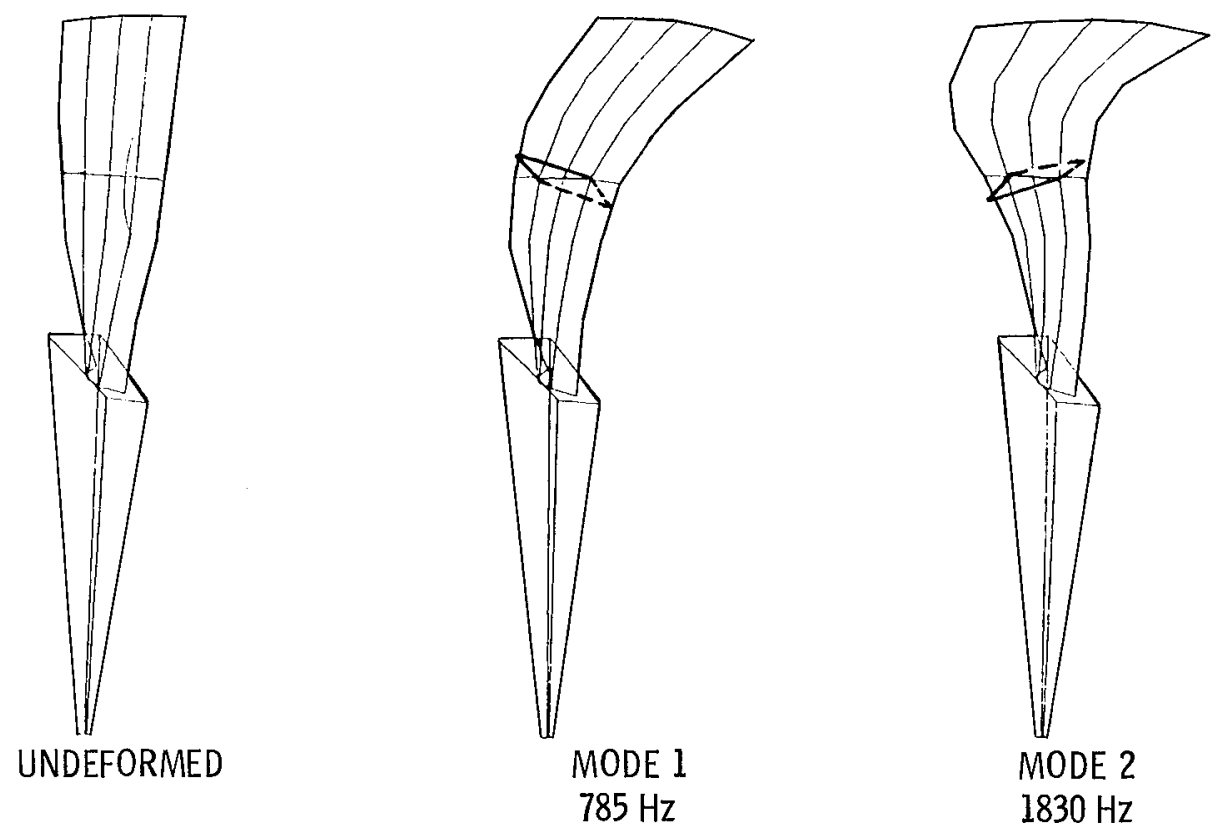

Figure 3. - Aeroelastic finite element analysis of bladed shrouded disks (bell code). 


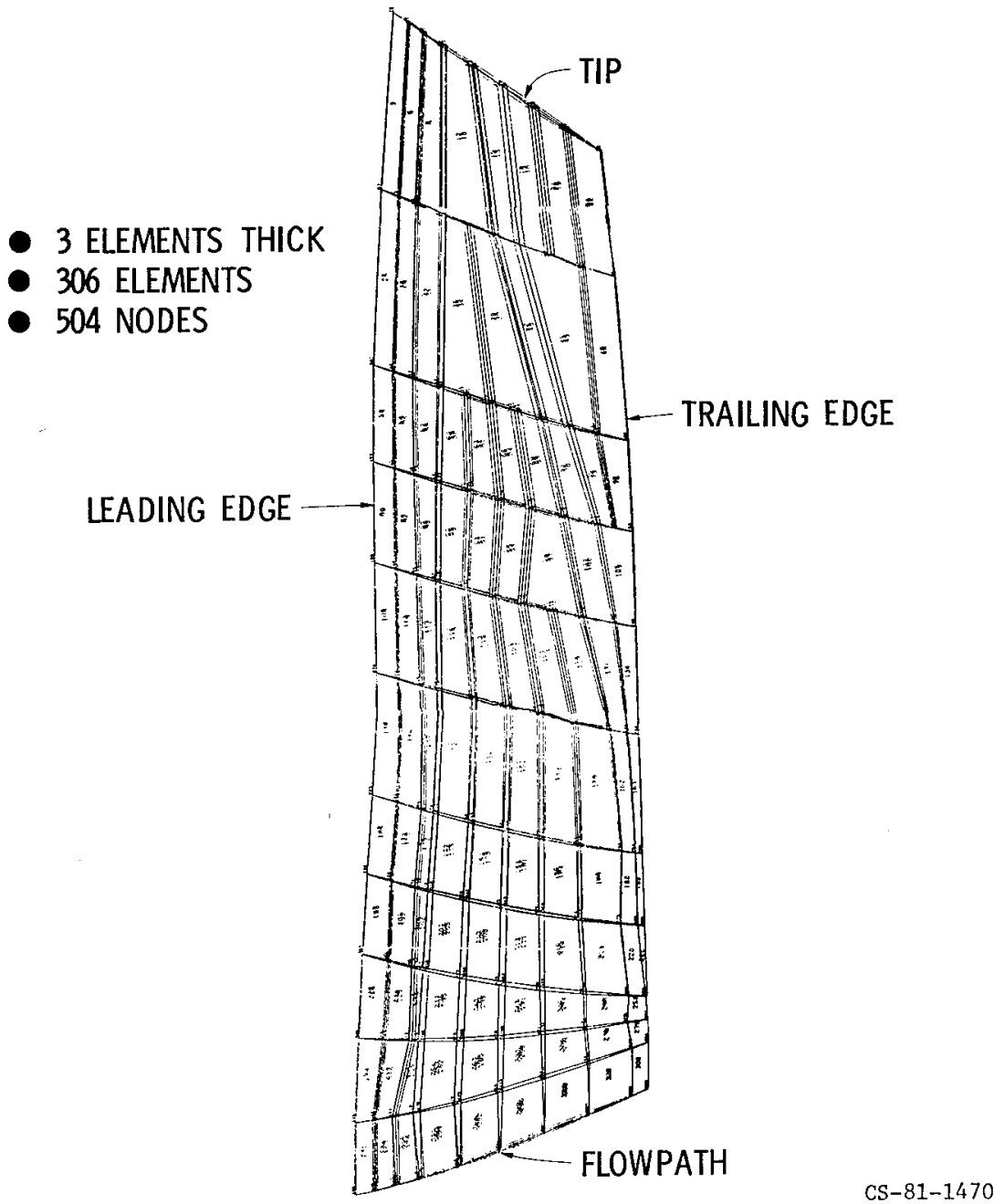

Figure 4. - Superhybrid composite blade finite element model. 


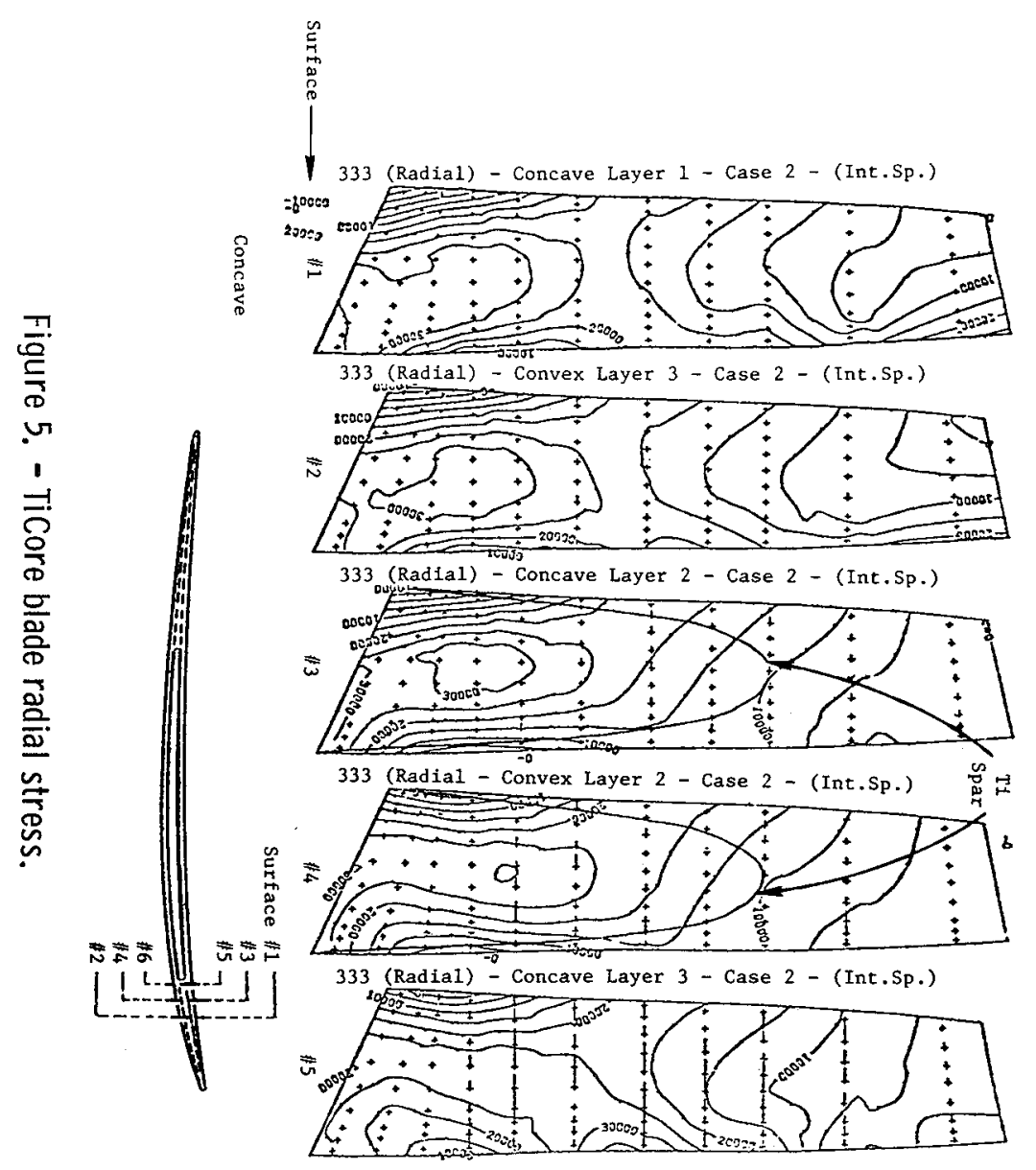




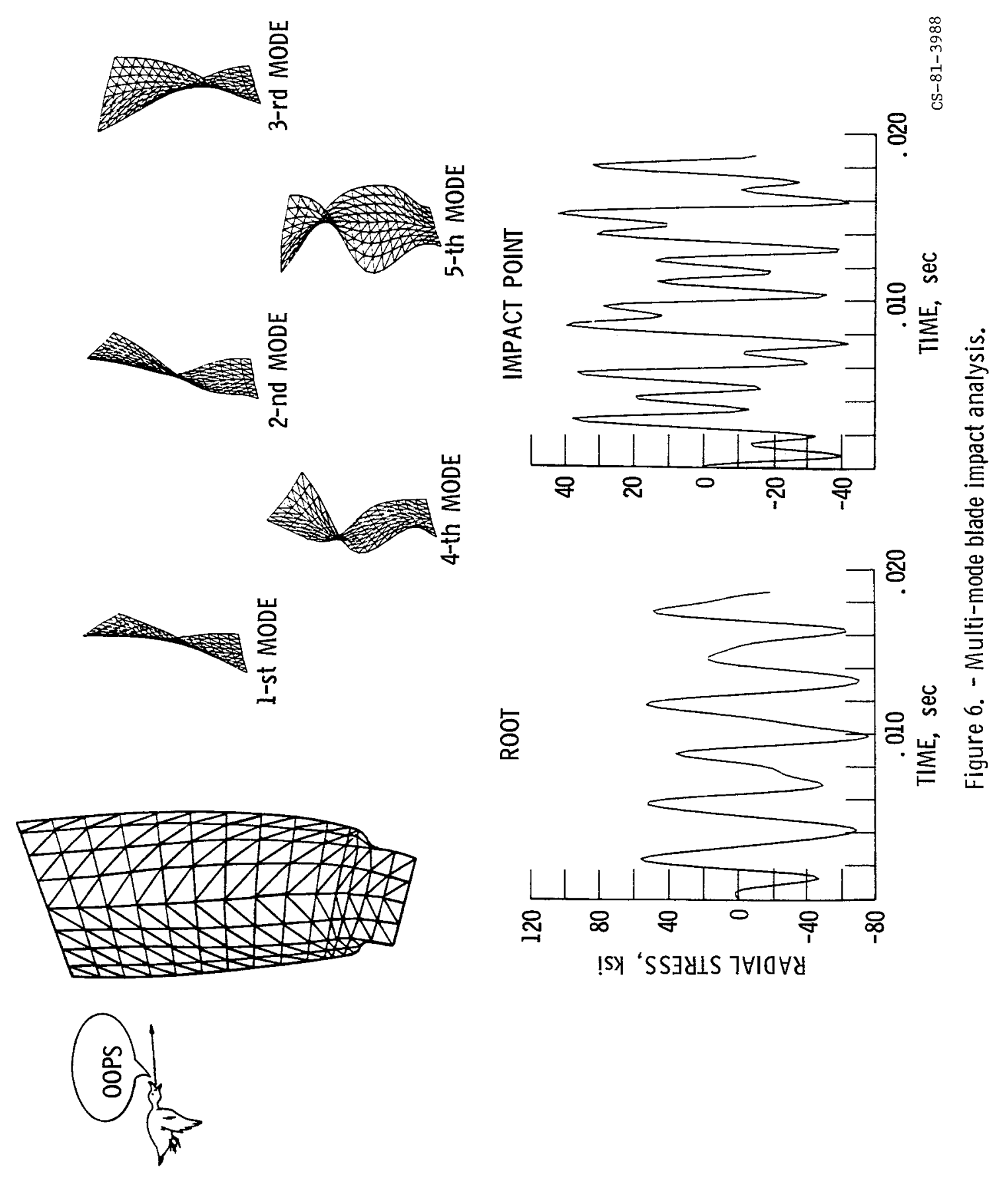






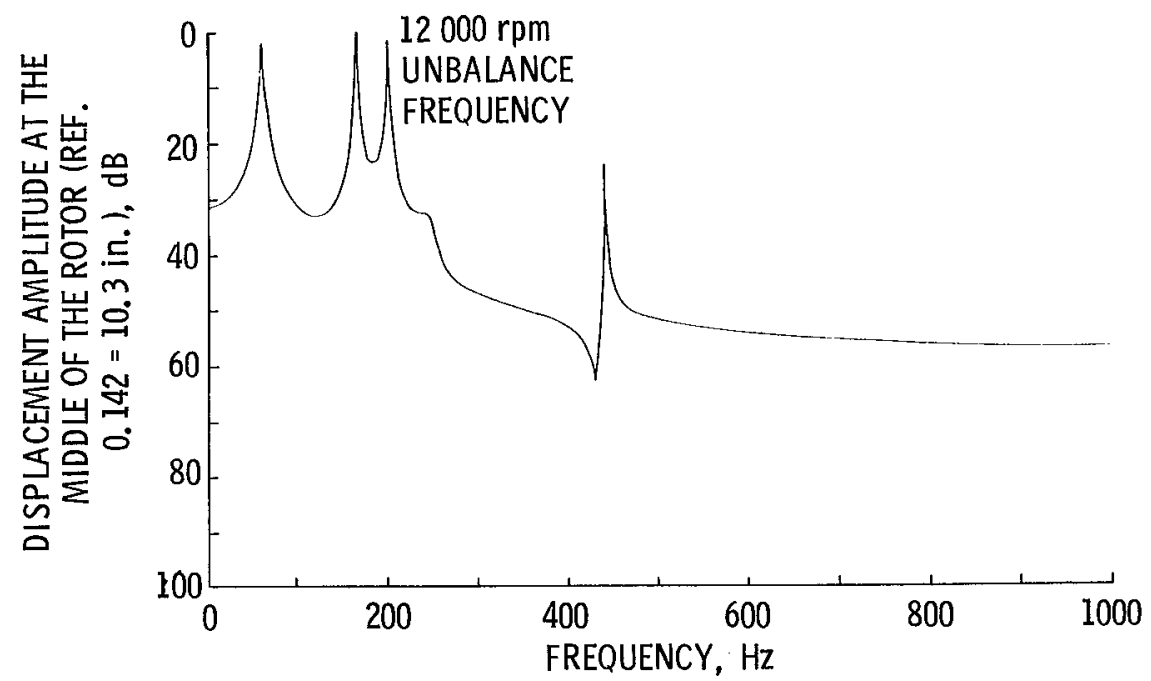

Figure 9. - Demonstrator model, $100 \mathrm{~g}$-in, sudden unbalance at the fan at $12000 \mathrm{rpm}$ (spectrum-log mag. versus linear frequency).
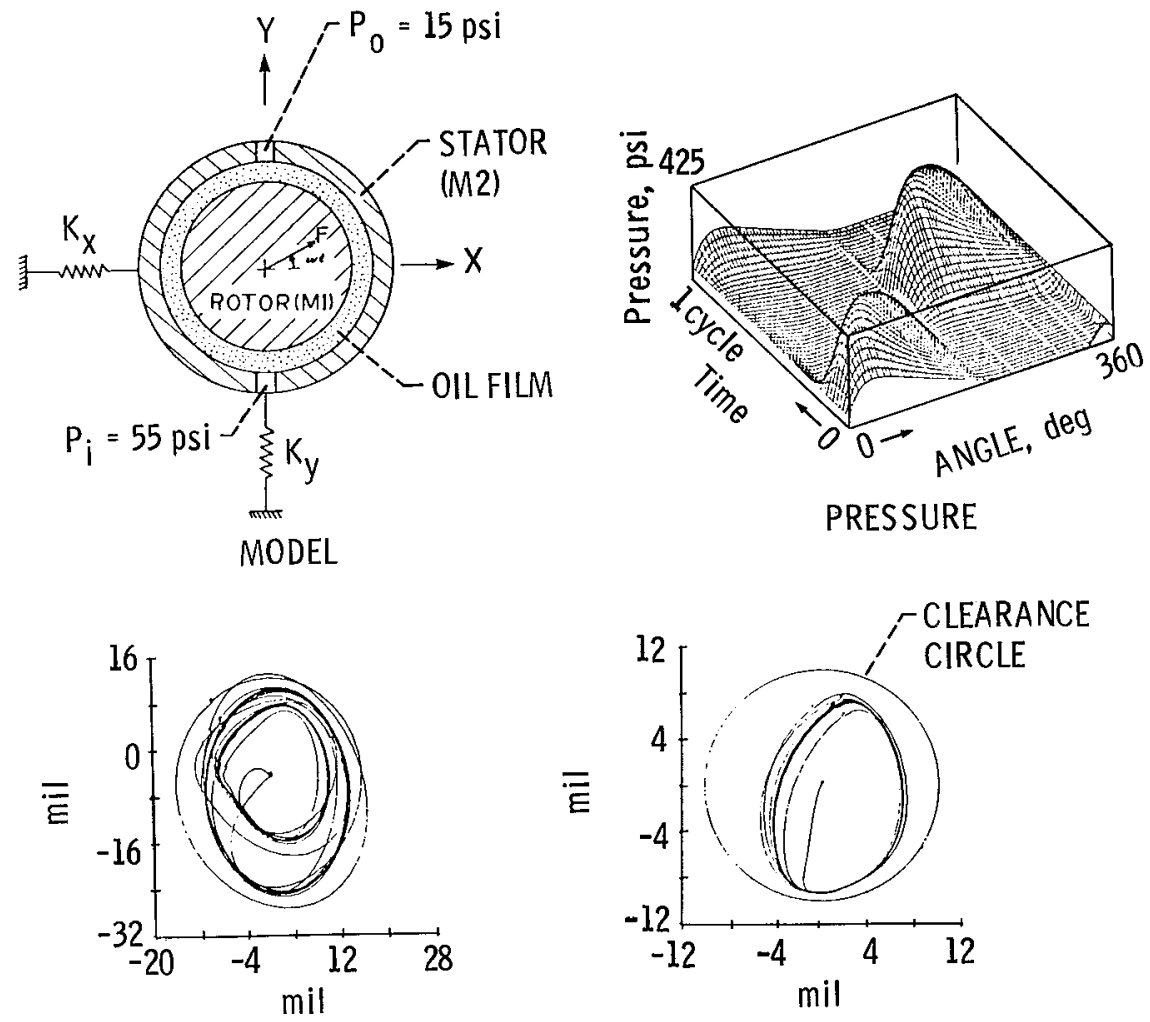

STATOR/ROTOR ORBITS

RELATIVE ROTOR ORBITS

Figure 10. - Bearing damper interactive element - pressure and orbit responses. 

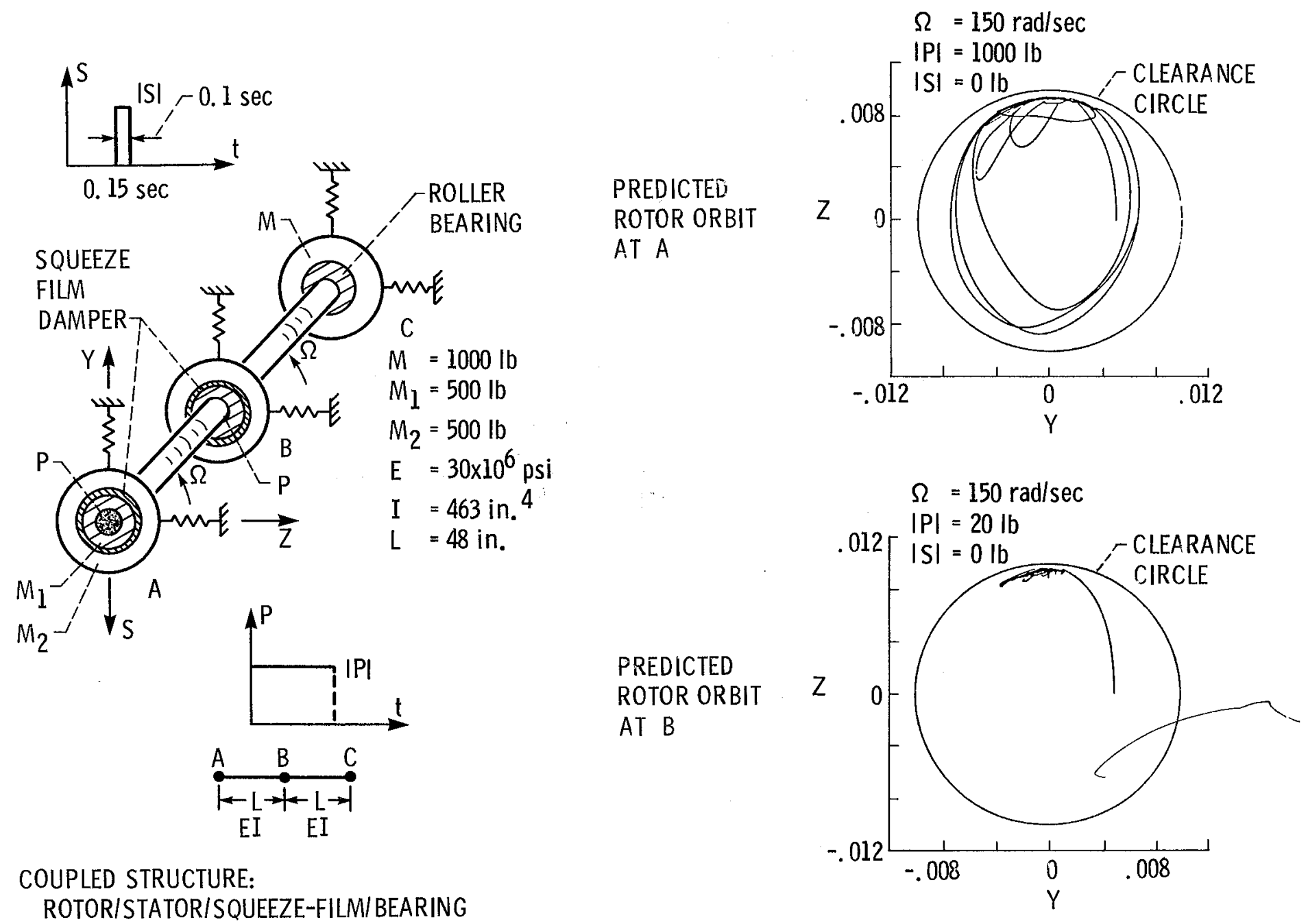

Figure 11. - Structural dynamics analysis using rotor/stator/squeeze-film/bearing interactive finite element. 


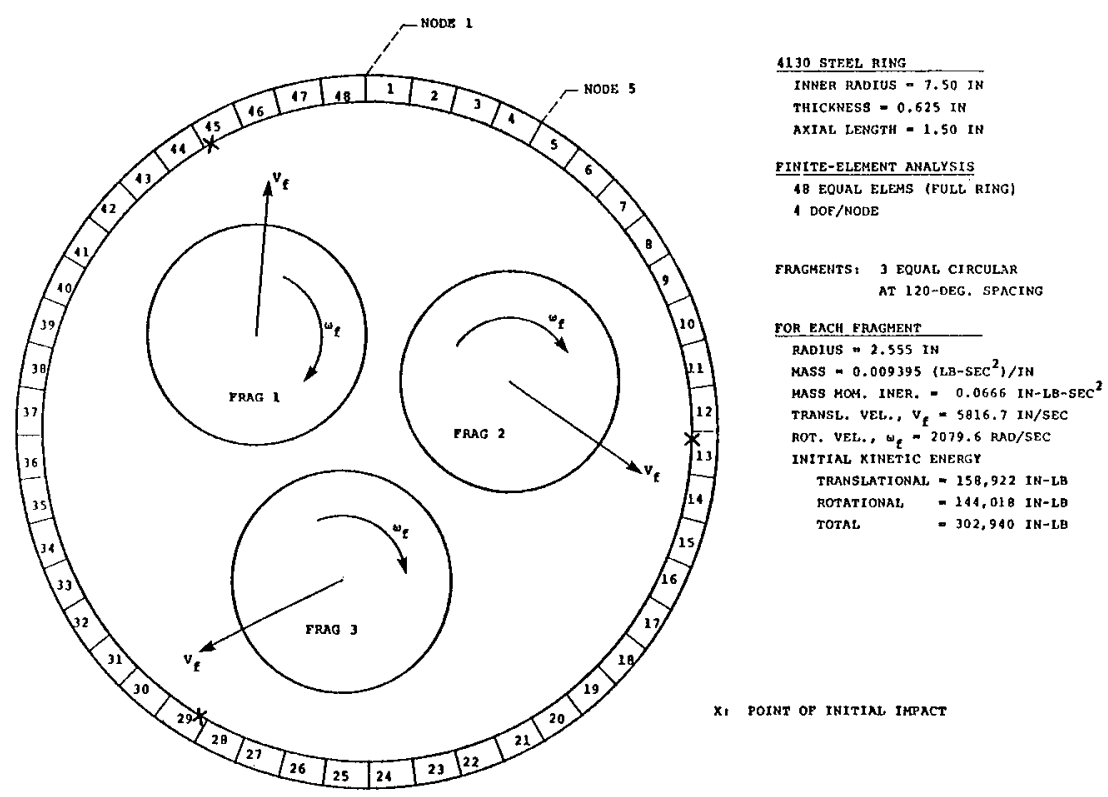

Figure 12. - Geometric, test, and modeling data for the 4130 steel containment ring subjected to tri-hub T58 rotor burst in NAPTC test 201.

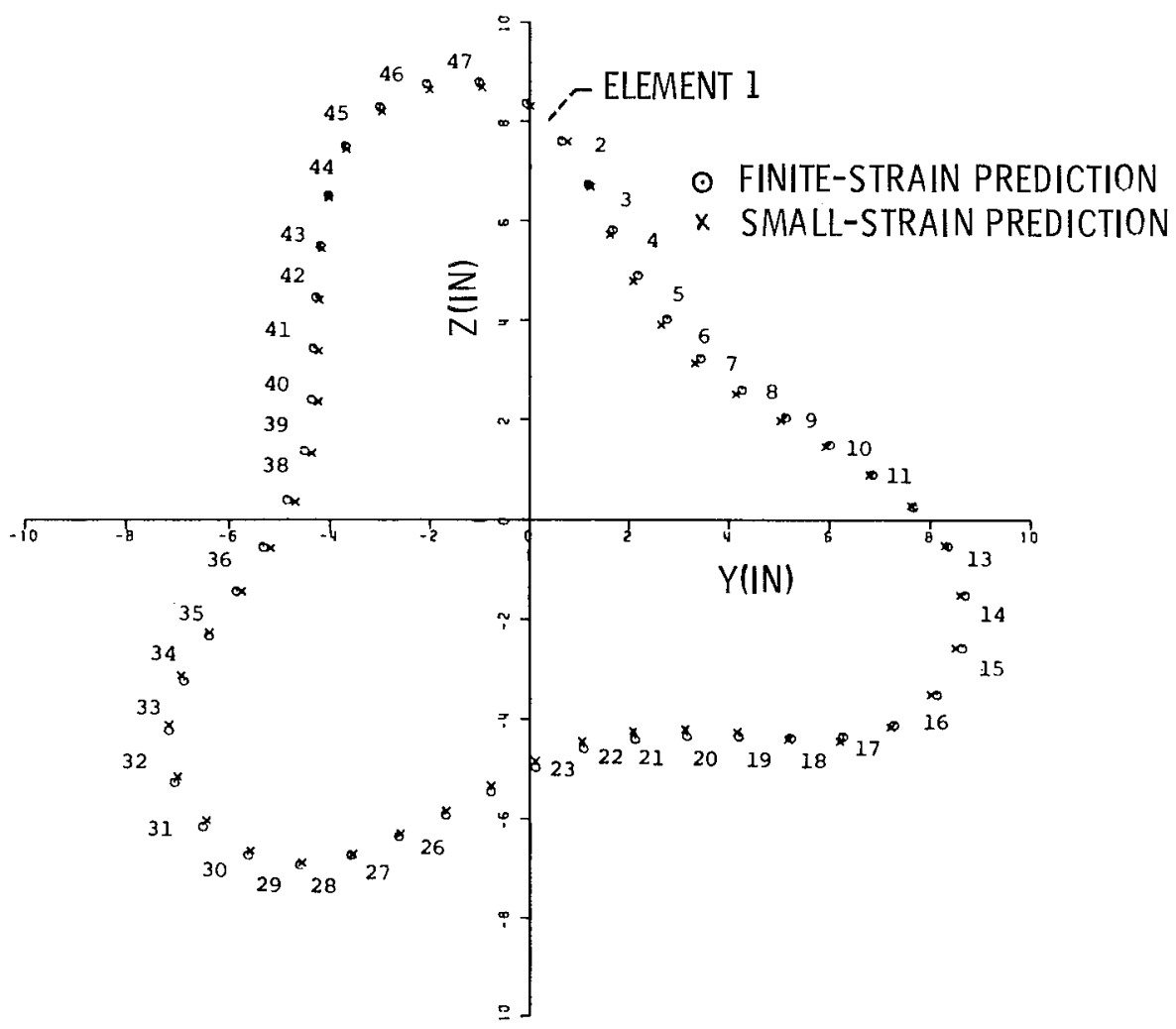

Figure 13. - Deformed configuration $1180 \mu \mathrm{sec}$ after burst. 

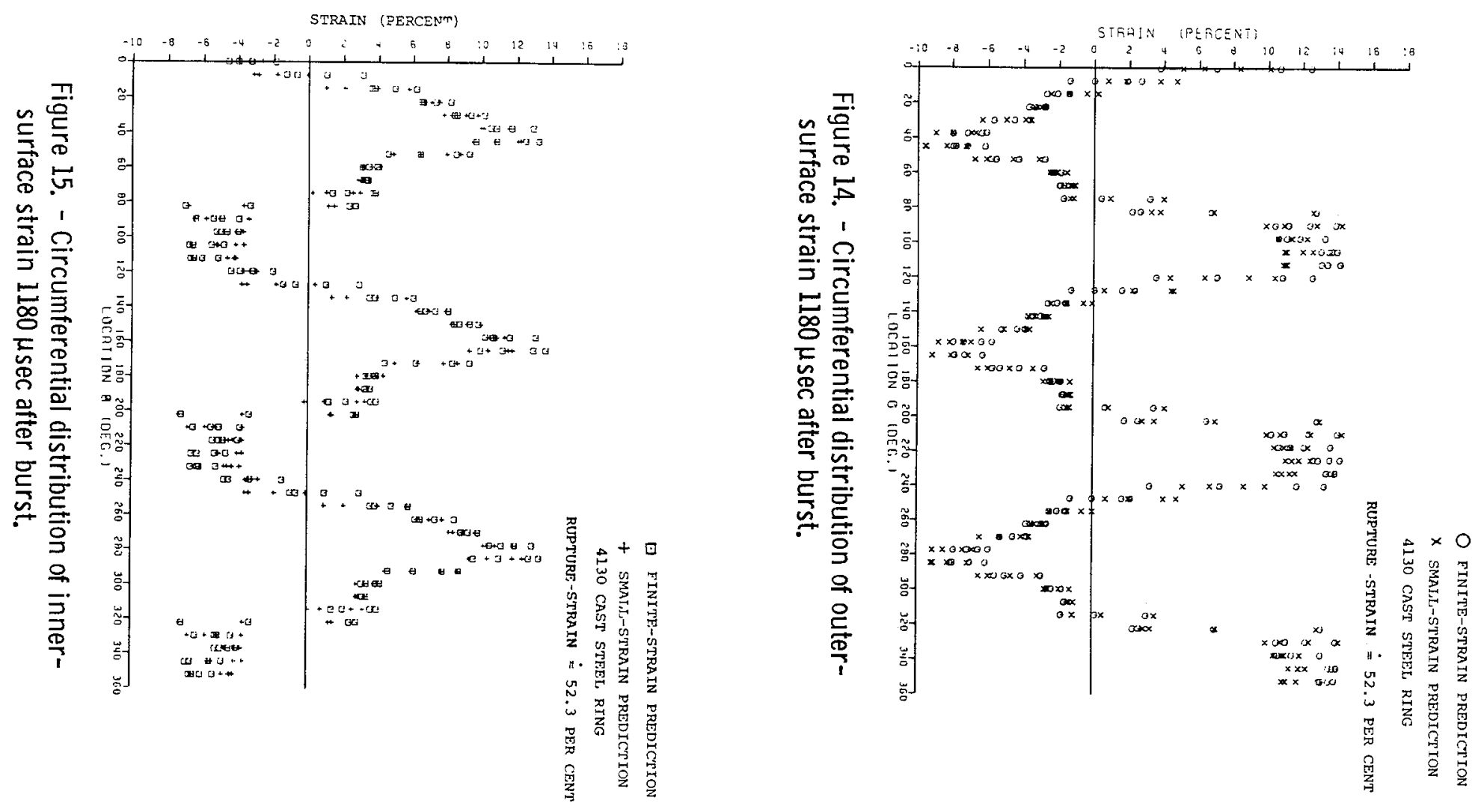


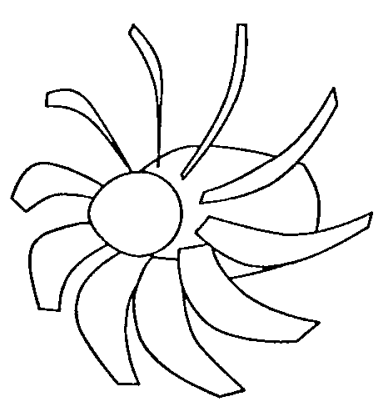

PROPERLIER STAGE

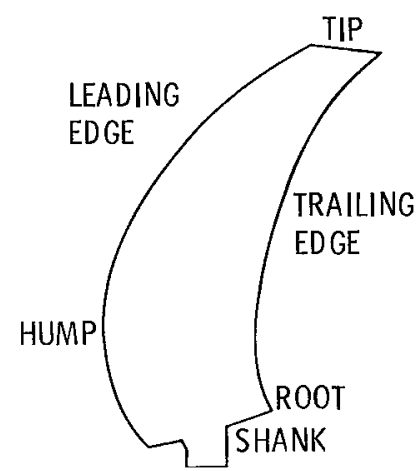

PROPELLER BLADE

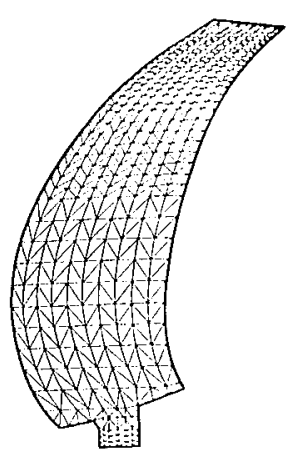

423 GRID POINTS, 744 ELEMENTS

Figure 16. - Turboprop stage propeller and finite element model.

SUMMARY OF TENSILE BUCKLING ROTOR SPEEDS

\begin{tabular}{|l|c|}
\hline \multicolumn{1}{|c|}{ TURBOPROP } & $\begin{array}{c}\text { ROTOR SPEED, } \\
\text { rpm }\end{array}$ \\
\hline $\begin{array}{l}\text { UNSWEPT- } \\
\text { COMPOSITE } \\
60^{\circ}-\text { SWEPT- } \\
\text { TITANIUM } \\
60^{\circ}-\begin{array}{c}\text { SWEPT- } \\
\text { COMPOSITE } \\
60^{\circ}-\text { SWEPT- } \\
\text { COMPOSITE } \\
\text { (STIFF } \pm 45^{\circ} \text { PLIES) }\end{array}\end{array}$ & 12470 \\
\hline
\end{tabular}

CAMPBELL DIAGRAM $60^{\circ}$ SWEPT-COMPOSITE TURBOPROP

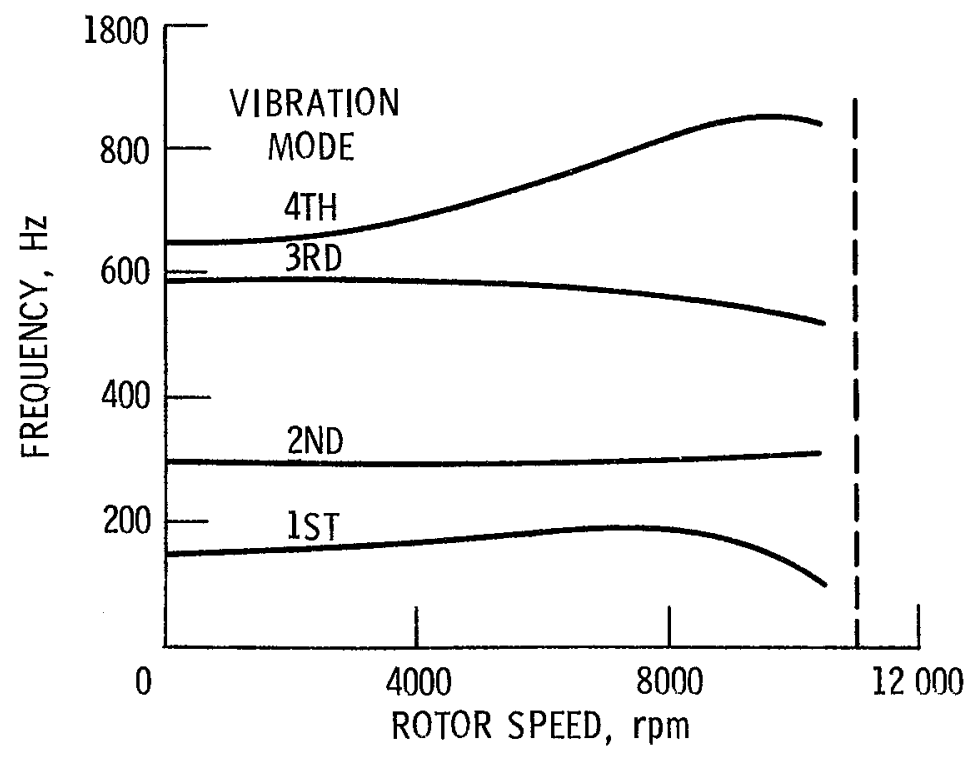

EFFECT OF ROTOR SPEED ON THE VIBRATION MODE I NODAL LINES, $60^{\circ}$ SWEPT COMPOSITE TURBOPROP

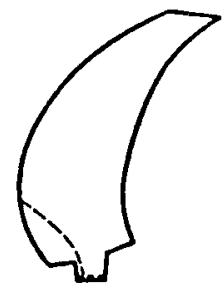

$0 \mathrm{rpm}$

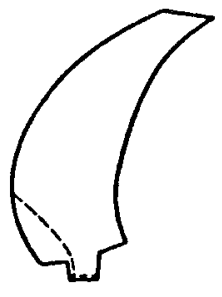

$3000 \mathrm{rpm}$

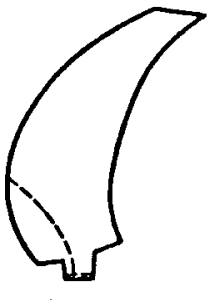

$6000 \mathrm{rpm}$

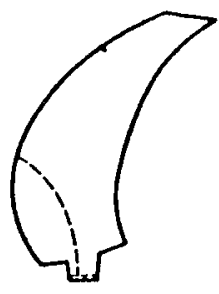

$7500 \mathrm{rpm}$

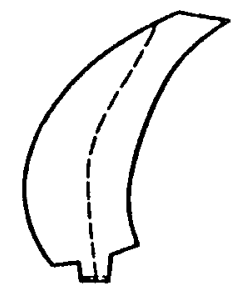

$9000 \mathrm{rpm}$

Figure 17. - Structural behavior of advanced turboprops. 


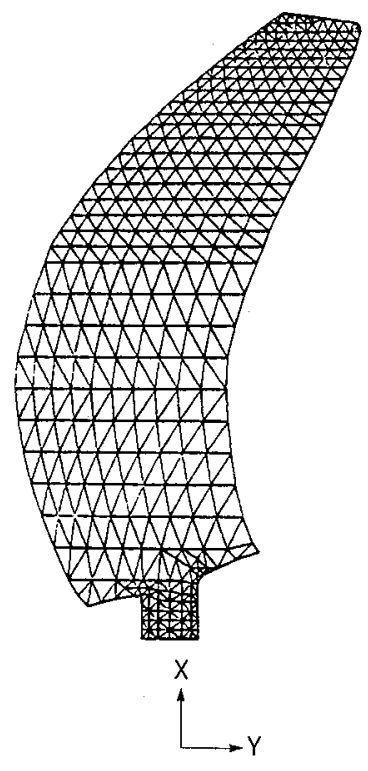

ANALYSIS MODEL

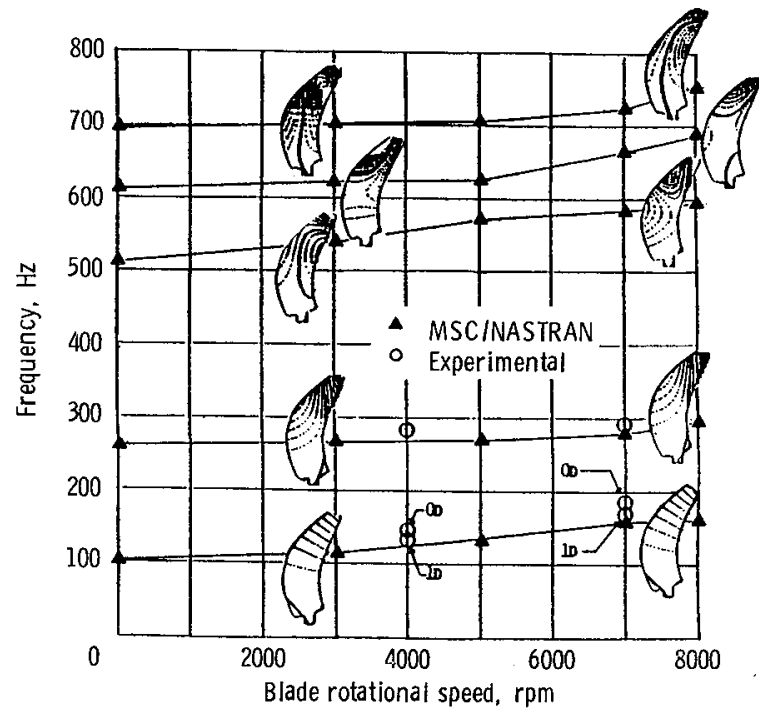

CAMPBELL DIAGRAM

Figure 18. - Composite turboprop vibration analysis. 


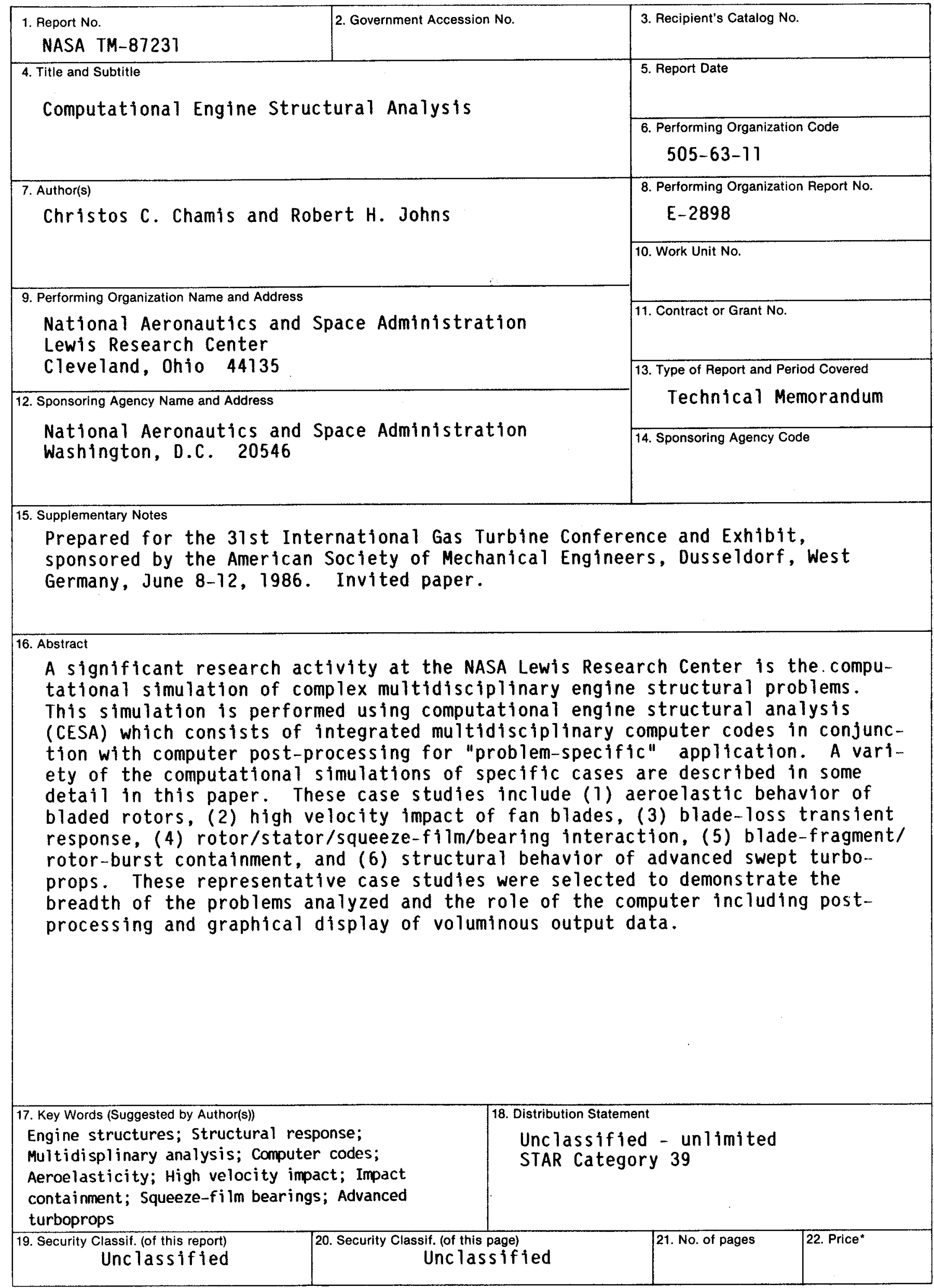

*For sale by the National Technical Information Service, Springfield, Virginia 22161 
